\title{
Flexible dispersal strategies in native and non-native ranges: environmental quality and the 'good-stay, bad-disperse' rule
}

\author{
Cang Hui, Núria Roura-Pascual, Lluís Brotons, Robert A. Robinson and Karl L. Evans \\ C. Hui (chui@sun.ac.za), Centre for Invasion Biology, Dept of Botany and Zoology, Stellenbosch Univ., Private Bag X1, Matieland 7602, South \\ Africa. - N. Roura-Pascual, Àrea de Zoologia, Dept de Ciències Ambientals, Fac. de Ciències, Univ. de Girona, Campus Montilivi, ES-17071 \\ Girona, Catalunya, Spain. NR-P also at: Centre Tecnològic Forestal de Catalunya (CTFC), Ctra. St. Llorenç de Morunys km 2, ES-25280 \\ Solsona, Catalonia, Spain. - L. Brotons, European Bird Census Council (EBCC) and Centre Tecnologic Forestal de Catalunya (CTFC), Ctra. \\ St. Llorenç de Morunys km 2, ES-25280 Solsona, Catalonia, Spain. - R. A. Robinson, British Trust for Ornithology, The Nunnery, Thetford, \\ Norfolk IP24 2PU, UK. - K. L. Evans, Dept of Animal and Plant Sciences, Univ. of Sheffeld, Sheffeld S10 2TN, UK.
}

\begin{abstract}
Dispersal strategies are one of the most important determinants of range dynamics and a surrogate for invasiveness. We tested three inter-related hypotheses derived from demographic and ecological models: $\left(\mathrm{H}_{1}\right)$ short-distance dispersal strategies arise at native range margins due to their demographic advantage; $\left(\mathrm{H}_{2}\right)$ in non-native areas a high diffusion rate is favoured at the advancing range front for niche filling; $\left(\mathrm{H}_{3}\right)$ environmental deterioration can increase dispersal and lead to a 'good-stay, bad-disperse' strategy. Spatially and temporally explicit rates of spread and dispersal kernels of the European starling Sturnus vulgaris were generated for its native range (Britain) using ringing records from 1909 to 2008, and for a non-native area (South Africa) using ringing data and distributional records since its introduction in 1897 . There was a marked spatial and temporal variation in the rate of spread within both native and non-native ranges. In the native range the rate of spread declined with increasing distance from the species' European distribution (contradicting $\mathrm{H}_{1}$ ). In the non-native range the rate of spread increased with distance from the introduction locality (supporting $\mathrm{H}_{2}$ ). The annual rate of spread in the native range also increased significantly when environmental conditions were deteriorating as indicated by marked population declines and relatively low abundance $\left(\mathrm{H}_{3}\right)$, providing clear evidence for flexible dispersal strategies based on a 'good-stay, bad-disperse' rule. Starlings' dispersal kernel followed an inverse power law and showed strong anisotropy and significant divergence between native and invasive populations, suggesting a flexible strategy comprising a dynamic response to spatial and temporal environmental variation with implications for predicting dispersal and range dynamics arising from environmental change.
\end{abstract}

Understanding the structure of species' geographic ranges and their dynamics, which reflect the interplay between its dispersal capacity, environmental tolerances and biotic interactions (Guisan and Thuiller 2005, Soberon 2007, Roura-Pascual et al. 2011), is central to biogeography and conservation (Whittaker et al. 2005). Dispersal strategy has been suggested to be a more important determinant of range dynamics than demographic factors, such as generation time and population growth rate (Vandenbosch et al. 1992, Caswell et al. 2003). Early research in this area focused on describing patterns and formulating hypotheses (Travis and Dytham 1999, Gyllenberg et al. 2002, Gros et al. 2006), with rather limited attention being paid to empirical testing of associated theory. We provide a rare example of simultaneous tests of multiple inter-related hypotheses concerning the spatial and temporal variation of species' dispersal strategies. We do so using contemporary and historic data on ringing recoveries and geographic distributions from paired native and non-native ranges that provide ideal natural experiments for unravelling the mechanisms and factors behind range dynamics (Broennimann et al. 2007).

Dispersal can be costly as it requires energy expenditure and will reduce survival and fecundity if the new environment is less favourable than the original. Conversely, dispersal can be beneficial as it can promote release from natural enemies and reduce intra-specific competition and inbreeding (Keane and Crawley 2002, Dytham 2009). This trade-off between costs and benefits is predicted to result in different dispersal strategies at the edges of native and non-native ranges. Assuming that native range margins are stable and imposed by unfavourable environments beyond the range margin, reduced dispersal rates and distances can result in a higher proportion of individuals staying in good quality habitats than those of poor quality (Pulliam 2000). In such situations niche conservatism (Wiens and Graham 2005, Crisp et al. 2009) will result in short-distance dispersal strategies as long-distance dispersal imposes too great a risk that individuals will arrive in unsuitable areas outside the 
species' geographic range (Travis and Dytham 1999, With et al. 1999, Gros et al. 2006). We thus expect that a shortdistance dispersal strategy will be exhibited at the edge of a species' native range (hypothesis I).

In non-native ranges a long-distance dispersal strategy is expected at the edge of the advancing front (Hughes et al. 2003, Simmons and Thomas 2004). This long-distance dispersal enables individuals to reduce the intensity of intra-specific competition and rapidly cross geographic and environmental barriers (Suarez et al. 2001, Nathan 2006, Phillips et al. 2006, Von der Lippe and Kowarik 2007), and thus has an advantage over other forms of dispersal (Higgins et al. 2003, Bowler and Benton 2005). In addition, spatial sorting of individuals with different dispersal abilities can also locate strong dispersers at the range front during the range expansion (Phillips et al. 2010, Shine et al. 2011). We thus expect that in non-native areas a longdistance dispersal strategy will occur at the advancing range front (hypothesis II). Hypotheses I and II currently lack sufficient support as they have been subject to surprisingly few empirical tests (Cody and Overton 1996, Phillips et al. 2006).

The dynamic nature of dispersal strategies (Williamson 2009) enables species to adjust these strategies in response to global and regional environmental change (Bowler and Benton 2005, Suter et al. 2009). Empirical tests of the association between environmental degradation and dispersal are rare. We expect that the interplay between environmental quality and species' life history will generate a 'good-stay, bad-disperse' strategy in which dispersal rates increase following a decline in environmental quality (hypothesis III; Hui et al. 2011). This resembles the strategy of 'win-stay, lose-shift' that outperforms other behavioural strategies in evolutionary game theory (Nowak and Sigmund 1993). More generally, since introduced species often experience rapid adaptation and niche shift in their novel environments (Broennimann et al. 2007, Prentis et al. 2008), a shift in dispersal strategies is expected between native and non-native ranges. This shift may arise from changes in the proportion of long-distance dispersal events as well as changes in dispersal distance. It can be difficult to disentangle the relative roles of these two factors, but quantifying the proportion of these low probability long-distance dispersal events could be vital in advancing the study of biological invasion (Blackburn et al. 2009).

Here, we test these three hypotheses using the European starling Sturnus vulgaris as a case study. This is an ideal study system as data pertaining to range dynamics over a hundred year period are available from both the native range margin (Britain, located at the north-west edge of the species' native range) and the non-native range (South Africa); moreover, starlings exhibit the anticipated flexibility in their behavioural and life history traits following establishment outside their native range (Sol et al. 2002, Evans et al. 2005, Hockey et al. 2005). Our analyses are hypothesis driven and thus represent a notable advance on previous descriptive studies of starling range expansion (Wing 1943, Kessel 1953, Flux and Flux 1981) which ignore the South African range, and do not assess spatially and temporally explicit dispersal strategies.

\section{Methods}

\section{Study species and data}

The European starling Sturnus vulgaris occurs naturally across Eurasia, and is one of the globe's most successful avian invaders (Lowe et al. 2004). Approximately $80 \%$ of introduced populations have become established (Sol et al. 2002), and the non-native range now includes North America, Australia, New Zealand and South Africa (Long 1981). In South Africa, the species' range is still expanding following the release of 18 birds in Cape Town in 1897 (Harrison et al. 1997).

The dispersal strategy of the native British population was estimated using ringing records from the British Trust for Ornithology (BTO), including recoveries of starlings across Britain from 1909 to 2008 . We used the records of all birds ringed and recovered between 15 April and 30 September within Britain $(n=13332)$ and thus restricted analyses to the British breeding population that is essentially sedentary and thus eliminated migratory movements from the analysis (Freeman et al. 2007). Ringing and recovery effort in Britain is broadly representative of the species' distribution and seems sufficiently high to limit spatial bias in the probability of detecting short and long-distance dispersal (Wernham et al. 2002). Indeed, ringing effort is sufficiently unbiased to enable a wide range of dispersal strategies to be identified (Paradis et al. 1998). We adopted a conservative approach, however, and re-sampled an equal number of records per site and per year to reduce any potential effects of spatial and temporal variation of ringing effort (see below).

Although the dispersal of European starlings in the native range has been quantified using ring re-encounter data (Paradis et al. 1998) its spatial and temporal variation has not been assessed; moreover, variation in re-encounter probabilities between native and non-native ranges prohibits a direct comparison (Korner-Nievergelt et al. 2010). In the nonnative South African range ringing records exhibit geographical biases that reflect the distribution of sampling effort; the 79 ringing records available from 1955 to 2004 are all located near Cape Town, the place of introduction, and have a maximum dispersal distance of only $43 \mathrm{~km}$ (SAFRING, Avian Demography Unit). We thus further used historical data describing the starling's distribution within South Africa (47 historical records of first occurrences from 1897 to 1982; Supplementary material Appendix 1) to calculate dispersal behaviour with regard to both the rate of spread and the dispersal kernel.

\section{Rate of spread}

The rate of spread $\left(v ; \mathrm{km} \mathrm{yr}^{-1}\right)$ describes the speed at which the range front is advancing. The asymptotic rate of spread can be estimated by the intrinsic population growth rate $\left(r ; \mathrm{yr}^{-1}\right)$ and the diffusion rate $\left(D ; \mathrm{km}^{2} \mathrm{yr}^{-1}\right)$ as $v=(4 r D)^{1 / 2}$ (Skellam 1951, Vandenbosch et al. 1992). That is, the rate of spread reflects both the capacity of dispersal and population growth. The diffusion rate $\left(\mathrm{km}^{2} \mathrm{yr}^{-1}\right)$ is an indicator of mobility without considering establishment and has been frequently used in dispersion models and measures the area 
occupied per unit time (Skellam 1951, Vandenbosch et al. 1992). Diffusion rates of 20,200 and $2000 \mathrm{~km}^{2} \mathrm{yr}^{-1}$ equate approximately to an annual movement radius of respectively $2.5,8$ and $25 \mathrm{~km}$. The diffusion rate can be estimated using ringing (i.e. mark-recapture) data according to the following formula (Turchin 1998, p. 258):

$$
D=\frac{\sum_{i=1}^{n} l_{i}^{2}}{4 \sum_{i=1}^{n} t_{i}}
$$

where $l_{i}$ is the distance between the ringing and recovery sites and $t_{i}$ is the duration of the period between these events.

We calculated the diffusion rate of starlings in Britain according to this formula using the BTO ringing records. As South African ringing data were limited to the vicinity of the introduction site we first estimated the rate of spread $(v=$ distance/duration) from historical distribution data (Supplementary material Appendix 1) and then derived the diffusion rate as $D=v^{2} /(4 r)$; this formula is exactly equivalent to the one above (Skellam 1951, Vandenbosch et al. 1992). The population intrinsic growth rate $(r)$ was calculated using Okubo's (1986) exponential model for the British population and Caswell's (2003) and Clark and Martin's (2007) matrix population model for the South African population, with the model parameters (including the annual survival rate, nesting attempts, clutch size and nest success) estimated from the literature (see Supplementary material Appendix 2 for details).

\section{Dispersal kernel}

The dispersal kernel measures the frequency distribution of individual dispersal distance and is a crucial determinant of species range dynamics (Caswell et al. 2003). Quantifying the form of the dispersal kernel is important as individual movements may not follow the Brownian random walk that leads to a Gaussian dispersal kernel and different forms entail different rates of spread (Nathan 2006). More than twenty forms of dispersal kernels have been reported in the literature (Jongejans et al. 2008, Hawkes 2009), but the negative exponential function $\left(f(d) \sim e^{-\alpha \cdot d}\right)$ and the inverse power function $\left(f(d) \sim d^{-a}\right)$ are particularly frequently used (Molofsky and Ferdy 2005, Fric and Konvicka 2007), and we thus use these forms in this study. Ringing records were used for producing dispersal kernels in both native and non-native ranges.

\section{Tests}

To test hypothesis I that short-distance dispersal strategies predominate at the edge of the species' native range, we calibrated the spatial realization of the rate of spread in Britain. Records were demarcated according to ringing localities, with 46 localities used in the analysis. Each locality contains $>100$ records with an average of 239 records per locality, and other locations are excluded due to insufficient data. To mitigate the effect of variable re-sighting effort across localities, we performed a re-sampling of 100 records per locality, without replacement, 10000 times.
The locality-specific median diffusion rates from the resampling were used for the calculation of the rate of spread and then for a natural neighbour interpolation (also termed area-stealing interpolation; Sibson 1981) with four added artificial boundary points to expand the interpolation across Britain (ArcGIS 9.2; ESRI). This method generates a smooth contour surface based on the local structure of input data and is robust against irregularly distributed data (Watson 1992).

Range margins of terrestrial species can arise either because habitat quality declines beyond the threshold at which the species can maintain viable populations, or when the species runs out of land. Starling densities are much lower at the western and northern edges of their British distribution (Gibbons et al. 1993) but coastal boundaries clearly play a major role in defining the edge of the species' geographic range in Britain. In such situations, it is preferable to measure the edge of species' British distributions as distance from the species' distribution in continental Europe (Blackburn et al. 1999), and we follow this approach here. Specifically, we used the minimum distance from the starling's distribution on the European continent as a metric of the distance from the range edge. We thus assessed the nature of the correlation between the rate of spread and the minimum distance to the starling's European continental distribution. Hypothesis I predicts a negative correlation as localities that are further from the continent are further from the centre of starling's geographic range.

To test hypothesis II that long-distance dispersal strategies should predominate at the advancing front of the non-native range, the same natural neighbour interpolation method was used to calibrate an equivalent map in South Africa using the rate of spread at 46 localities estimated from the historical records (Supplementary material Appendix 1). We then calculated the correlation between the rate of spread and the distance to the original introduction site, Cape Town; hypothesis II predicts a positive correlation.

To test hypothesis III that dispersal will increase when environmental conditions deteriorate, we first performed a re-sampling of 100 records from 10000 simulations to mitigate potential effects of annual variation in ringing effort and then used the median values from the re-samplings to first calculate a moving average annual diffusion rate $\left(D_{M}=\left(D_{t}+D_{t+1}+D_{t-1}\right) / 3\right)$ and then transform it into the rate of spread in the native range in order to reduce excessive temporal variation. Years with fewer than 100 recoveries were not included in the analysis. Following previous work, we used annual estimates of the starling's abundance index as an indicator of habitat quality (Chamberlain et al. 2000, Freeman et al. 2007) and correlated it with the moving average annual rate of spread, with a negative correlation expected from hypothesis III. The abundance index is available from 1966 and is calculated by the British Trust for Ornithology's annual monitoring of breeding population size using a combination of territory mapping and line transects and indicates a population decline of over $80 \%$ (Baillie et al. 2010). Empirical studies have conclusively linked this decline to deteriorating habitat quality, especially in agricultural areas (Robinson and Sutherland 2002, BirdLife International 2004, Newton 2004, Robinson et al. 2005, Baillie et al. 2010). 
To further test whether dispersal strategy differs between native and non-native ranges, we compared diffusion rates in the native and non-native ranges. The rate of spread was not directly used here for comparison because the population growth rate in Britain is different from the rate in South Africa, and thus the comparison of the rate of spread does not solely reflect the difference in dispersal strategy. Diffusion rates were compared in two ways: 1) a re-sampling of 100 records for 10000 simulations for the native population to compare with the diffusion rates estimated for the two-phase range expansion from the 47 historic records in South Africa (Supplementary material Appendix 1); 2) a re-sampling of 50 records for 10000 simulations for short-distance movements of $43 \mathrm{~km}$ or less in the native range ( $\mathrm{n}=12136$ records) to facilitate the comparison with the diffusion rate calculated from the same re-sampling method for the 79 ringing records in South Africa (with a maximum dispersal distance of $43 \mathrm{~km}$ ). A lack of overlap in $83 \%$ confidence intervals represents a significant difference in diffusion rates at $\alpha=0.05$ (note that a lack of overlap in $95 \%$ confidence intervals indicates a statistically significant difference at much smaller thresholds of $\alpha$; Payton et al. 2003).

To further assess the source of the potential spatial and temporal variation in diffusion rates, we compared the shortdistance dispersal kernel (distance $\leq 43 \mathrm{~km}, \mathrm{n}=12136$ ) in Britain with the dispersal kernel calculated from the 79 ringing records in South Africa. Since the movement of birds could be anisotropic, we further calculated the dispersal kernels for six different directions $\left(0 \sim 60^{\circ}, 60 \sim 120^{\circ}, 120 \sim 180^{\circ}\right.$, $\left.180 \sim 240^{\circ}, 240 \sim 300^{\circ}, 300 \sim 360^{\circ}\right)$ in the native range. We used the Akaike information criterion corrected for small sample size ( AIC $_{c}$ ) to examine which form provides the most parsimonious fit to the dispersal kernels. We used a two-sample Kolmogorov-Smirnov (KS) test to examine the difference between dispersal kernels (Conover 1998) by calculating a dimension-free distance, $D_{F}=D_{K S} \sqrt{n_{1} n_{2} /\left(n_{1}+n_{2}\right)}$, where $D_{K S}$ is the $K S$ distance, $n_{1}$ and $n_{2}$ the number of records to generate the two dispersal kernels, and the critical value for rejecting the null hypothesis that the two dispersal kernels are derived from the same probability distribution is $D_{F}>1.36(\mathrm{p}<0.05)$.

\section{Results}

There was a marked spatial variation in the rate of spread within the British native range (Fig. 1A). The rate of spread was typically low in western and central England and typically highest in northern and eastern coastal areas, with 95\% confidence interval ranging from 2.25 to $13.5 \mathrm{~km} \mathrm{yr}^{-1}$. There was a significant positive correlation between the rate of spread and the minimum distance from the starling's European distribution $(r=0.44, p<0.01)$. The rate of spread also varied spatially within the non-native South African range (Fig. 1B). It tended to be low $\left(<4 \mathrm{~km} \mathrm{yr}^{-1}\right)$ in areas surrounding mountainous barriers and high $\left(>16 \mathrm{~km} \mathrm{yr}^{-1}\right)$ at the landward edge of the species' current range, reflecting a slow rate of spread pre-1940 (6.1 $\left.\mathrm{km} \mathrm{yr}^{-1}\right)$ and a fast rate post-1940 (25.7 $\mathrm{km} \mathrm{yr}^{-1}$; Supplementary material Appendix 1, Fig. A1). The rate of spread was positively correlated with distance from the point of introduction $(r=0.46, \mathrm{p}<0.01)$.

The moving average annual rate of spread for dispersal in the native range was calculated for the $40 \mathrm{yr}$ for which over 100 recoveries were available per year (1951-1990). A linear model $\left(\mathrm{AIC}_{\mathrm{c}}=3.49\right.$, weight $\left.w=0.04\right)$ indicates that the rate of spread increases significantly by $0.106 \mathrm{~km} \mathrm{yr}^{-1}$ per annum $\left(\mathrm{r}=0.78, \mathrm{~F}_{1,38}=57.9, \mathrm{p}<0.01\right)$. A quadratic model provides a better fit to the data $\left(\mathrm{AIC}_{\mathrm{c}}=-3.01\right.$, weight $w=0.96$ ) and has a turning point in 1958 (Fig. 2A). Linear regressions around this break point indicated that there was no significant decline in the rate of spread between 1951 and $1958\left(\mathrm{~F}_{1,6}=2.83, \mathrm{p}=0.14\right)$, but a substantial increase after this period $\left(0.153 \mathrm{~km} \mathrm{yr}^{-1}\right.$ per annum, $\mathrm{F}_{1,30}=82.8$, $\mathrm{p}<0.01)$. The moving average annual rate of spread increases as the national abundance index declines (an increment of $-0.55 \mathrm{~km} \mathrm{yr}^{-1}$ per reduction in the abundance index of 0.1 ; $\mathrm{r}=-0.701, \mathrm{~F}_{1,23}=22.2, \mathrm{p}<0.01 ;$ Fig. 2B).
(A)

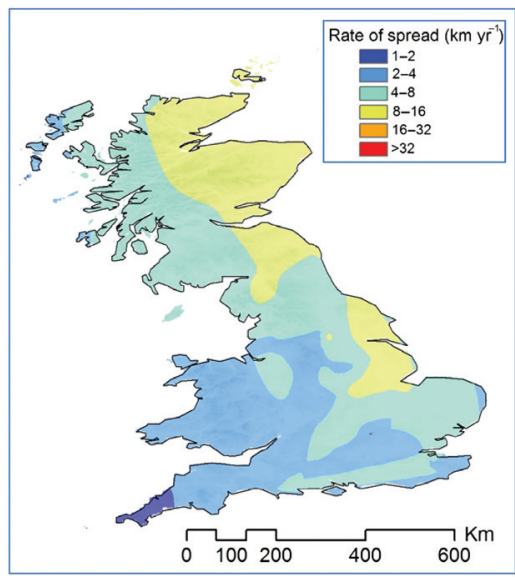

\section{(B) South Africa (non-native range)}

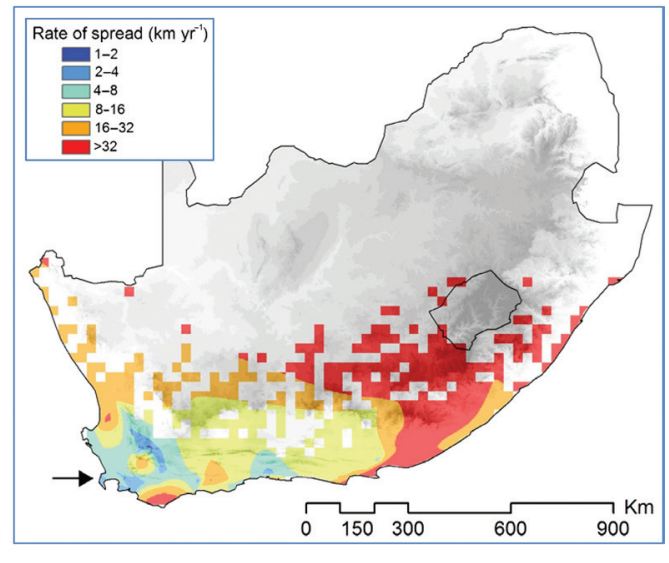

Figure 1. Spatially explicit rate of spread of European starling in (A) native and (B) non-native ranges, extrapolated by natural neighbour interpolation. In panel (B) the arrow indicates the introduction site (Cape Town, 1897) and the coloured grid indicate the starling's distribution at a quarter-degree resolution (Harrison et al. 1997). 

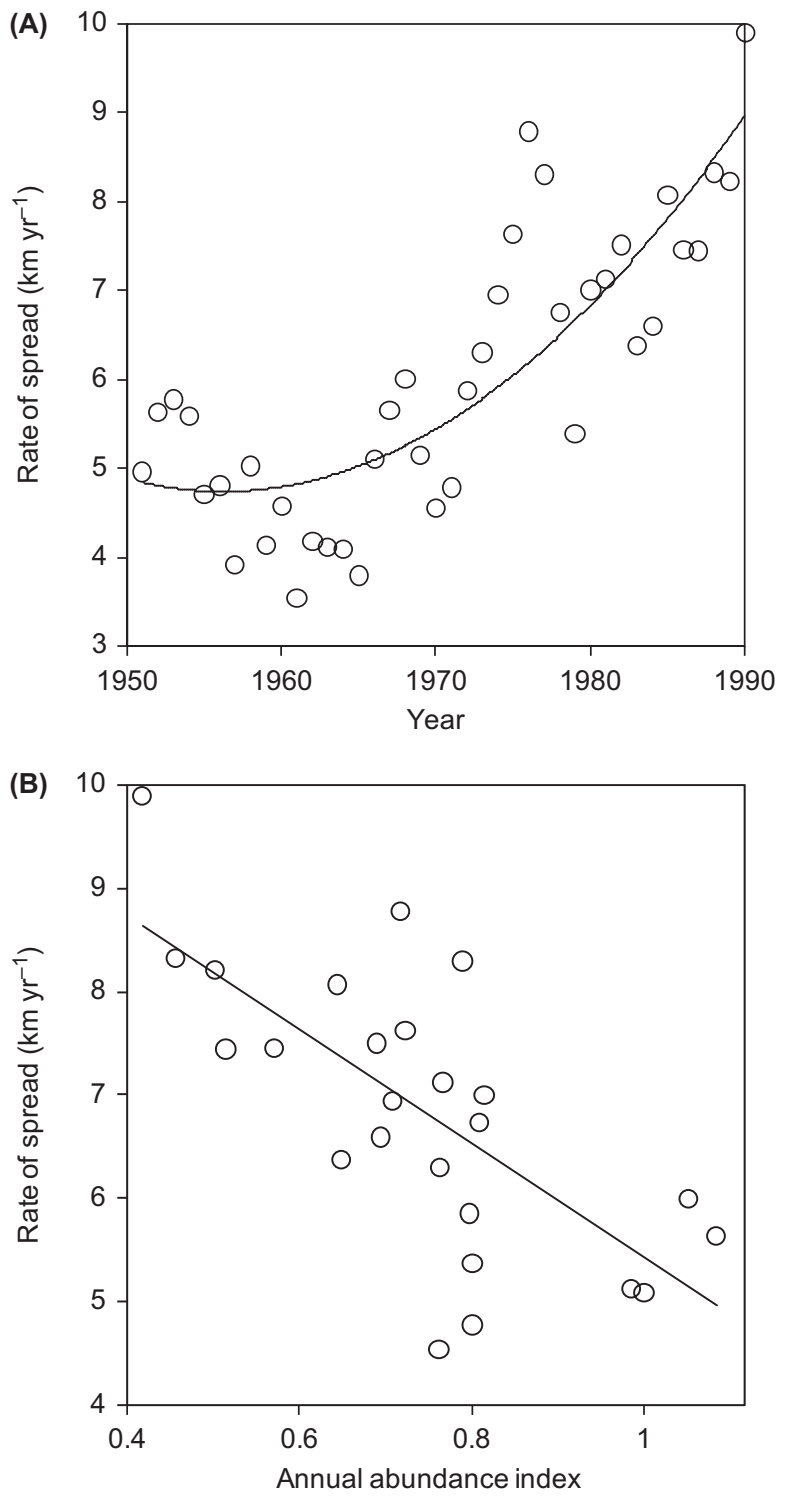

Figure 2. (A) Temporal dynamics of the moving average annual rate of spread (unit: $\mathrm{km} \mathrm{yr}^{-1}$ ) in the native range from 1951 to 1990; the curve represents the fit of a quadratic model. (B) The moving average annual rate of spread as a function of the abundance index, low values of which indicate reduced habitat quality (Baillie et al. 2010).

When only comparing the dispersal strategy, the median diffusion rate in the native range was $205 \mathrm{~km}^{2} \mathrm{yr}^{-1}$, with $83 \%$ confidence interval of 70 to $553 \mathrm{~km}^{2} \mathrm{yr}^{-1}$. This confidence interval overlapped that of the diffusion rates estimated from the historical records pre-1940 in the non-native range (35.5-142.4 $\mathrm{km}^{2} \mathrm{yr}^{-1}$ ) but was lower than the $83 \%$ confidence interval of the non-native diffusion rates post1940 (1034-1915 $\mathrm{km}^{2} \mathrm{yr}^{-1}$; Fig. 3A). Diffusion rates in the non-native range calculated using ringing records estimated a median of $13 \mathrm{~km}^{2} \mathrm{yr}^{-1}$ (83\% confidence interval: 8.6-16.4 $\mathrm{km}^{2} \mathrm{yr}^{-1}$ ), and overlapped the $83 \%$ confidence intervals of the rate of short-distance dispersal in the native range (5.9$19.8 \mathrm{~km}^{2} \mathrm{yr}^{-1}$; median $11.6 \mathrm{~km}^{2} \mathrm{yr}^{-1}$; Fig. 3B). This overlap indicates the lack of a significant difference at $\alpha=0.05$ (Payton et al. 2003).

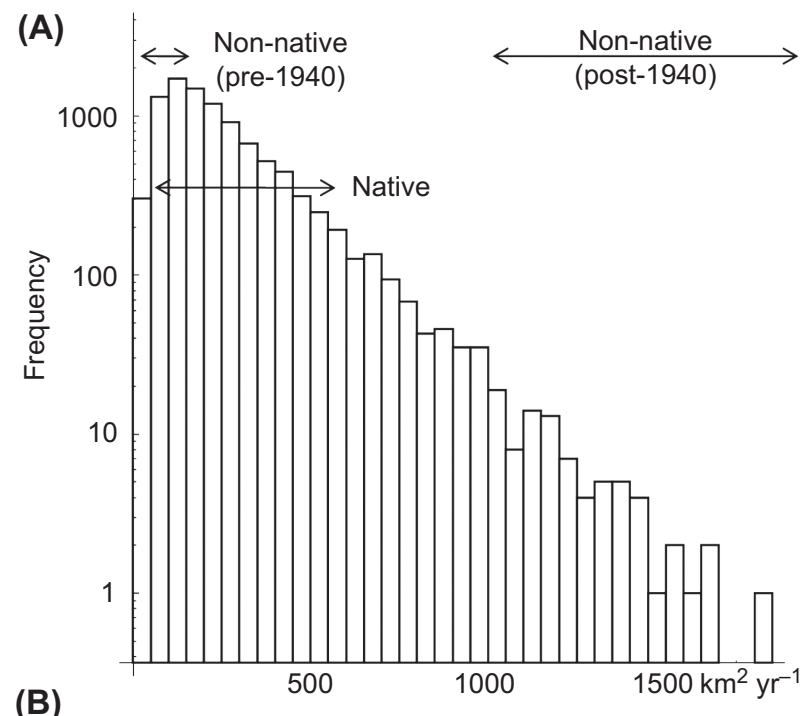

(B)

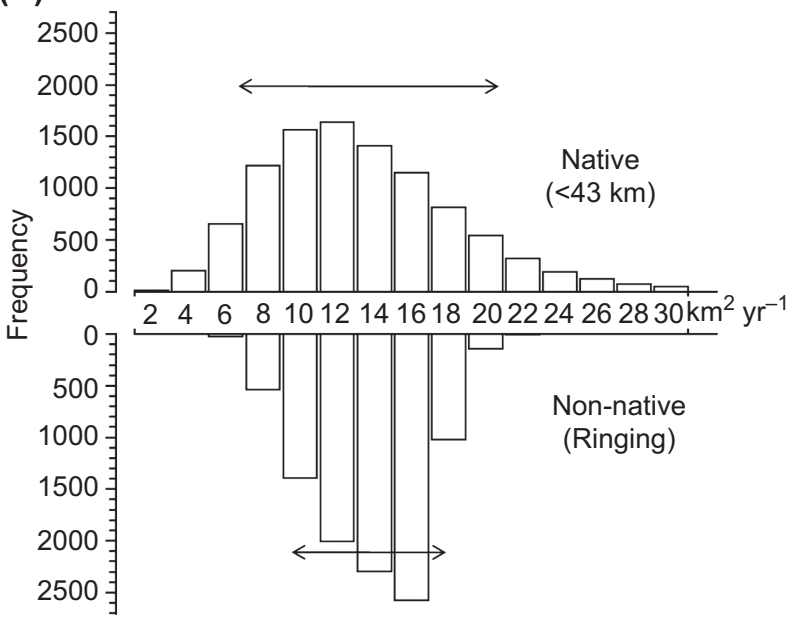

Figure 3. (A) Histogram of the diffusion rates in the native range from 10000 re-samplings of 100 records, with comparative estimates from the historical data in the non-native range. (B) Histograms of the diffusion rates in the native range from short-distance records $(\leq 43 \mathrm{~km})$ and in the non-native range from ringing records. Double-headed arrows indicate $83 \%$ confidence intervals of the diffusion rate.

Dispersal kernels followed the form of an inverse power function (Fig. 4A). Specifically, the short-distance dispersal kernel $(\leq 43 \mathrm{~km})$ in the native range fitted an inverse power function $\left(y=1.12 x^{-1.49}, \mathrm{R}^{2}=0.97, \mathrm{AIC}_{\mathrm{c}}=-143.6\right.$, weight $w>0.999)$ much better than a negative exponential model $\left(\mathrm{R}^{2}=0.82, \mathrm{AIC}_{\mathrm{c}}=-97.2\right.$, weight $\left.w<0.001\right)$; so did the dispersal kernel in the non-native range calculated using the ringing records (inverse power function: $y=0.93 x^{-1.24}$, $\mathrm{R}^{2}=0.8, \quad \mathrm{AIC}_{\mathrm{c}}=-145.3$, weight $w=0.981 ;$ negative exponential model: $\mathrm{AIC}_{\mathrm{c}}=-137.4$, weight $w=0.019$; Fig. 4B). Furthermore, the native dispersal kernel was significantly steeper than the non-native dispersal kernel $\left(D_{F}=2.34, \mathrm{p}<0.05\right.$; Fig. 4B). The dispersal kernels in Britain were anisotropic in all six directions, with a minimum $D_{F}=1.43(\mathrm{p}<0.05)$; however, the overall relationship between diffusion rates and the exponent of dispersal kernels in different directions was not significant $(\mathrm{r}=0.68$, $\mathrm{p}=0.14 ;$ Fig. 5). 

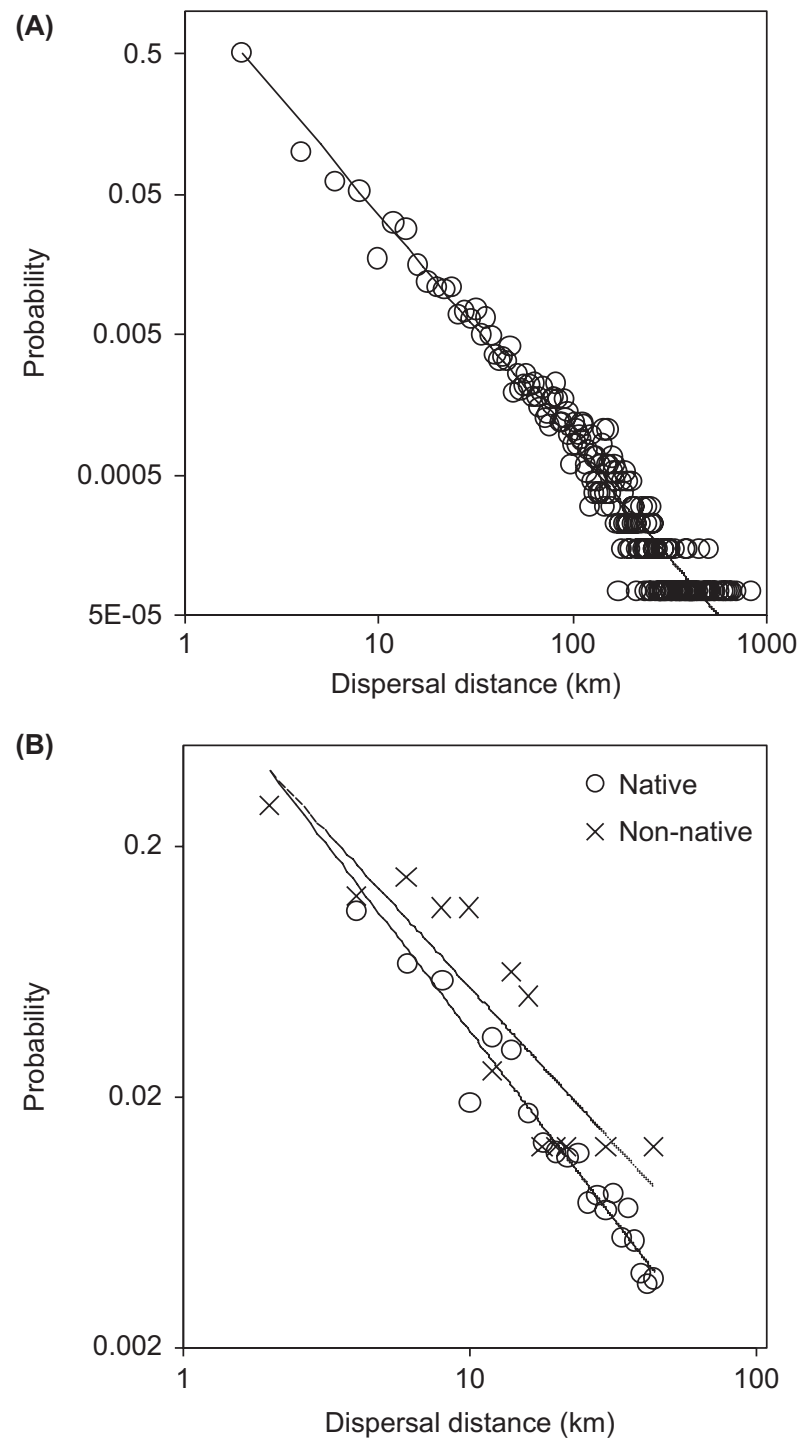

Figure 4. Inverse power functions of (A) the dispersal kernel for all movements within Britain during the breeding season and (B) dispersal kernels for short-distance records $(\leq 43 \mathrm{~km})$ in Britain (solid line) and ringing records in South Africa (dashed line). All dispersal kernels are produced using a 2-km distance class; that is, all records are binned to the dispersal distance $(d)$ class of $\leq 2 \mathrm{~km}, 2<d \leq 4$ $\mathrm{km}, 4<d \leq 6 \mathrm{~km}$ etc.

\section{Discussion}

The European starling exhibits high spatial and temporal variation in its rate of spread in both its native and nonnative geographic ranges. This suggests that starlings may be able to adjust dispersal strategies in response to environmental conditions, such as habitat quality and landscape topography. The mechanisms generating this spatial variation are unknown and plasticity seems likely to play an important role. Genetic adaptation could also contribute, however, as whilst gene-flow can limit evolutionary capacity at range margins strong selection pressures can result in genetic divergence arising even when gene flow is high (Senar et al. 2006). Starling populations at the expanding edge of the species' non-native distribution also experience surprisingly limited

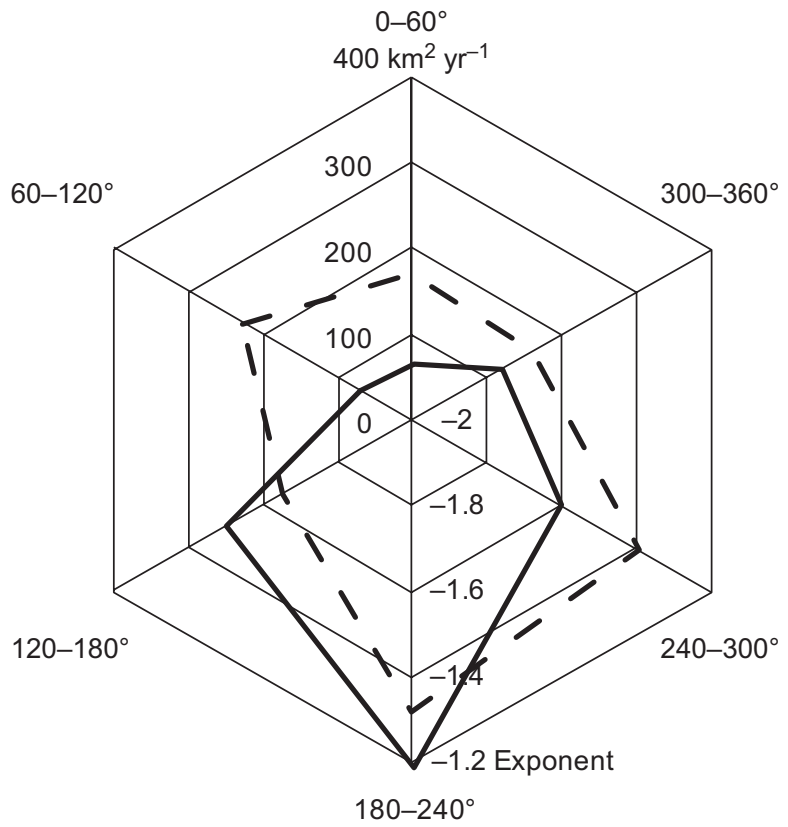

Figure 5. The median values of the diffusion rates (solid line) and the exponent of the inverse power function dispersal kernels (dashed line) in Britain, in six directions: $0 \sim 60^{\circ}, 60 \sim 120^{\circ}, 120 \sim 180^{\circ}$, $180 \sim 240^{\circ}, 240 \sim 300^{\circ}$ and $300 \sim 360^{\circ}$.

gene flow from other populations (Rollins et al. 2011). Despite the marked spatial variation in rates of spread we found little support for hypothesis I that short-distance dispersal strategies predominate at the edge of the species' native range (Gros et al. 2006). Greater temporal variation in habitat quality at the range margin, relative to the range centre, could promote dispersal and contribute to the lack of evidence for hypothesis I (McPeek and Holt 1992, Holt 2003). Moreover, greater spatial variation in habitat quality at the range margin, compared to the range centre, could promote greater dispersal if high quality habitats were within achievable dispersal distances typical of the focal species. In addition, birds are active dispersers that may be able to avoid areas of low quality habitat, thus reducing selective pressures to exhibit short distance dispersal at range edges. Shortdistance dispersal strategies seem more likely to evolve in species that predominately disperse passively, such as plants whose seeds are dispersed by wind (Gros et al. 2006), and such species can exhibit variation in their dispersal capacity in response to changing environmental conditions (Imbert and Ronce 2001). Clearly, future studies of dispersal strategies at range margins should consider the interplay of multiple factors (Dytham 2009) and the dispersal mode of focal species.

The rate of spread in the South African non-native range provides empirical support for hypothesis II that invasive species at the niche-filling stage should exhibit an accelerating range front (Phillips et al. 2006, 2008). Spatial sorting could be one factor contributing to this pattern, with individuals with strong dispersal capacities being more likely to be present at the expanding range front (Shine et al. 2011). The twophase spread of starlings, consisting of a lag phase followed by a high velocity of range expansion, has also been reported 
in the North American non-native range (Okubo 1986), with $11.2 \mathrm{~km} \mathrm{yr}^{-1}$ pre-1915 and $51.2 \mathrm{~km} \mathrm{yr}^{-1}$ post-1915, which is double the velocity in South Africa $\left(6.1 \mathrm{~km} \mathrm{yr}^{-1}\right.$ pre-1940 and $25.7 \mathrm{~km} \mathrm{yr}^{-1}$ post-1940; Supplementary material Appendix 1). The greater velocity in North America does not appear to be caused by a difference in the diffusion rate (e.g. the post-1915 diffusion rate in North America, $2920 \mathrm{~km}^{2} \mathrm{yr}^{-1}$, lies within the $95 \%$ confidence interval of the post-1940 diffusion rate in South Africa, 417.3 3628.4 $\mathrm{km}^{2} \mathrm{yr}^{-1}$ ), rather it appears to be driven by a higher intrinsic rate of increase in North America $\left(r=0.224 \mathrm{yr}^{-1}\right)$ than in South Africa $\left(r=0.115 \mathrm{yr}^{-1}\right.$; Supplementary material Appendix 2) which is perhaps a consequence of more second broods and larger clutch sizes in the northern hemisphere compared to equivalent southern latitudes with different climatic regimes (Lack 1968, Cody 1971, Evans et al. 2005; Supplementary material Appendix 2). If correct, this suggests that range dynamics can be affected by physiological responses to environmental conditions, such as drier, warmer and less seasonal climates in South Africa compared to the starling's native range.

Theoretical studies of geographical range expansion frequently assume invariable diffusion rates and thus predict constant rates of spread (Skellam 1951, Vandenbosch et al. 1990, Holmes et al. 1994). This is correct for a stable environment but seems unlikely to apply to much of the globe which is experiencing rapid rates of environmental change that can alter species' dispersal abilities and other life-history traits (Dawson et al. 2011, Schoener 2011). Indeed, the 'win-stay, lose-shift' behavioural rule frequently emerges in the evolutionary game theory (Nowak and Sigmund 1993), and our data provide rare empirical evidence for an equivalent ecological strategy of 'good-stay, bad-disperse'. The annual diffusion rate in Britain varies by an order of magnitude between the 1950s and the late 1980s, equating to a threefold difference in the velocity of range expansion (Fig. 2A). Increased dispersal during periods of population decline and relatively low abundance (Fig. 2B), provide support for a 'good-stay, bad-disperse' behavioural strategy. Predictions of changes in spatial spread and rates of distributional change thus seem likely to be compromised if they ignore inherent fundamental uncertainties concerning spatial, temporal and environmental variation (Melbourne and Hastings 2009, Dawson et al. 2011). Our results not only demonstrate the strong spatial and temporal variability in dispersal rate (Fig. 1,2), but also reveal potential patterns and predictors of this dispersal variability that may be useful for reducing the uncertainty for predicting range dynamics under changing environmental conditions, such as those arising from climate change.

Native and non-native starling populations do not differ in the diffusion rate of their short-distance dispersal events; however, when considering all dispersal movements, diffusion rates are much greater during the period of non-native range expansion, i.e. post 1940, than in the native range (Fig. 3). This suggests that starlings' dispersal strategy comprises two components, a conservative short-distance dispersal component, which is expected to arise through niche conservatism, and a dynamic long-distance dispersal component. This flexibility presumably drives much of the spatial and temporal variation in starlings' diffusion rates and rate of spread, and it seems likely that most species with flexible dispersal patterns will exhibit dispersal strategies with conservative and dynamic components. Whilst the short-distance dispersal kernel is a truncated version of the full dispersal kernel we find that the form of the full dispersal kernel is conservative across the complete range of dispersal distances (i.e. there is no evidence for non-linearity at the log-log scale; Fig. 4A). Consequently, when data are limited, it appears feasible to extrapolate from the short-distance dispersal kernel to estimate the proportion of birds that disperse larger distances. Such extrapolations should, however, be used with extreme caution as the dynamic component of dispersal kernels in species with flexible dispersal strategies implies that there can be much variation in the form of dispersal kernels towards the distribution's tail.

The dispersal kernel of starlings strictly obeyed an inverse power function, $f(d) \sim d^{-\beta} \quad(\beta=1.5$; Fig. 4$)$, instead of a negative exponential function. The power-law dispersal kernel suggests that starlings do not follow Brownian random walk; rather, their movement resembles a Lévy flight (walk) and thus can generate stratified spread with the occupied areas forming a fractal clustered set of points (Shlesinger 2001), which is evident in the spread of starlings in North America (Kessel 1953). A power-law dispersal kernel of Lévy flight has also been found for animal foraging (Viswanathan et al. 1996, Ramos-Fernandez et al. 2004) and seed dispersal by wind (Nathan et al. 2002), as well as human travel (Brockmann et al. 2006). Because the exponent $\beta$ is less than two, the expected dispersal distance $\left(\int d \times f(d)\right)$ is unbounded, indicating a fat-tailed long-distance dispersal of starlings. This long-distance dispersal strategy could well explain the two-phase range expansion of starlings and other invasive species (Shigesada et al. 1995, Crooks 2005, Pyšek and Hulme 2005, Fric and Konvicka 2007, Floerl et al. 2009, Lyons and Scheibling 2009). Further research in dispersal ecology should strive to quantify the adaptive advantage of a power-law dispersal kernel over other forms (e.g. the negative exponential function), as well as the environmental and evolutionary conditions under which a power-law kernel is favoured.

In summary, we found no support for the widely assumed hypothesis that short-distance dispersal strategies evolve at native range margins, which may partly be a consequence of the increased flexibility of active dispersers compared to species with more passive dispersal strategies. We provide strong evidence, however, for flexible dispersal strategies that facilitate a dynamic response to spatial and temporal environmental variation. Dispersal in the non-native range increases with distance from the introduction site. In the native range increased dispersal is associated with deteriorating environmental conditions, which has consequences for modelling species' responses to environmental change.

Acknowledgements - We are grateful to Robert D. Holt and Melodie A. McGeoch for discussion during the Niche Evolution Conference held at Zürich, Switzerland in 2009, and to subcommittee B fund at Stellenbosch Univ. and the NRF Blue Skies and Incentive Programme for financial support. NR-P benefited from a Beatriu de Pinós Postdoctoral Grant 2008 BP-B 00042 from the Catalan Agency for Management of Univ. and Research Grants (Generalitat de Catalunya). KLE is supported by the Leverhulme Trust. Mikko 
Mönkkönen provided constructive comments. The British and Irish Ringing Scheme is operated by the British Trust for Ornithology (BTO) with funding also from the Joint Nature Conservation Committee (JNCC), the National Parks and Wildlife Service (Ireland) and the ringers themselves, while the BTO operates the Breeding Bird Survey in partnership with the JNCC and the Royal Society for the Protection of Birds.

\section{References}

Baillie, S. R. et al. 2010. Breeding birds in the wider countryside: their conservation status 2009. - BTO Research Report No. 541, British Trust for Ornithology.

BirdLife International 2004. Birds in Europe: population estimates, trends and conservation status. - BirdLife International.

Blackburn, T. M. et al. 1999. Do local abundance of British birds change with proximity to range edge? - J. Biogeogr. 26: 493-505.

Blackburn, T. M. et al. 2009. Avian invasions: the ecology and evolution of exotic birds. - Oxford Univ. Press.

Bowler, D. E. and Benton, T. G. 2005. Causes and consequences of animal dispersal strategies: relating individual behaviour to spatial dynamics. - Biol. Rev. 80: 205-225.

Brockmann, D. et al. 2006. The scaling laws of human travel. - Nature 439: 462-465.

Broennimann, O. et al. 2007. Evidence of climatic niche shift during biological invasion. - Ecol. Lett. 10: 701-709.

Caswell, H. et al. 2003. Demography and dispersal: life table response experiments for invasion speed. - Ecology 84: 1968-1978.

Chamberlain, D. E. et al. 2000. Changes in the abundance of farmlands birds in relation to the timing of agricultural intensification in England and Wales. - J. Appl. Ecol. 37: 771-788.

Clark, M. E. and Martin, T. E. 2007. Modeling tradeoffs in avian life history traits and consequences for population growth. - Ecol. Model. 209: 110-120.

Cody, M. L. 1971. Ecological aspects of reproduction. - In: Farner, D. S. and King, J. R. (eds), Avian biology. Vol. 1. Academic Press, pp. 461-512.

Cody, M. L. and Overton, J. M. 1996. Short-term evolution of reduced dispersal in island plant populations. - J. Ecol. 84: 53-61.

Conover, W. J. 1998. Practical nonparametric statistics, 3rd ed. - Wiley.

Crisp, M. D. et al. 2009. Phylogenetic biome conservatism on a global scale. - Nature 458: 754-756.

Crooks, J. A. 2005. Lag times and exotic species: the ecology and management of biological invasions in slow-motion. - Ecoscience 12: 316-329.

Dawson, T. P. et al. 2011. Beyond predictions: biodiversity conservation in a changing climate. - Science 332: 53-58.

Dytham, C. 2009. Evolved dispersal strategies at range margins. - Proc. R. Soc. B 276: 1407-1413.

Evans, K. L. et al. 2005. Investigating geographic variation in clutch size using a natural experiment. - Funct. Ecol. 19: 616-624.

Floerl, O. et al. 2009. The importance of transport hubs in stepping-stone invasions. - J. Appl. Ecol. 46: 37-45.

Flux, J. E. C. and Flux, M. M. 1981. Population dynamics and age structure of starlings (Sturnus vulgaris) in New Zealand. - N. Z. J. Ecol. 4: 65-72.

Freeman, S. N. et al. 2007. Changing demography and population decline in the common starling Sturnus vulgaris: a multisite approach to integrated population monitoring. - Ibis 149: 587-596.
Fric, Z. and Konvicka, M. 2007. Dispersal kernels of butterflies: power-law functions are invariant to marking frequency. - Basic Appl. Ecol. 8: 377-386.

Gibbons, D. W. et al. 1993. The new atlas of breeding birds in Britain and Ireland: 1988-1991. - Poyser.

Gros, A. et al. 2006. Evolution of local adaptations in dispersal strategies. - Oikos 114: 544-552.

Guisan, A. and Thuiller, W. 2005. Predicting species distribution: offering more than simple habitat models. - Ecol. Lett. 8: 993-1009.

Gyllenberg, M. et al. 2002. Evolutionary suicide and evolution of dispersal in structured metapopulations. - J. Math. Biol. 45: 79-105.

Harrison, J. A. et al. 1997. The atlas of southern African birds. - BirdLife South Africa.

Hawkes, C. 2009. Linking movement behaviour, dispersal and population processes: is individual variation a key? - J. Anim. Ecol. 78: 894-906.

Higgins, S. I. et al. 2003. Are long-distance dispersal events in plants usually caused by nonstandard means of dispersal? - Ecology 84: 1945-1956.

Hockey, P. A. R. et al. 2005. Roberts birds of southern Africa, 7th ed. - John Voelker Bird Book Fund.

Holmes, E. E. et al. 1994. Partial-differential equations in ecology - spatial interactions and population dynamics. - Ecology 75: 17-29.

Holt, R. D. 2003. On the evolutionary ecology of species' range. - Evol. Ecol. Res. 5: 159-178.

Hughes, C. L. et al. 2003. Evolutionary trade-offs between reproduction and dispersal in populations at expanding range boundaries. - Proc. R. Soc. B 270: S147-S150.

Hui, C. et al. 2011. Modelling spread in invasion ecology: a synthesis. - In: Richardson, D. M. (ed.), Fifty years of invasion ecology: the legacy of Charles Elton. Wiley-Blackwell, pp. 329-343.

Imbert, E. and Ronce, O. 2001. Phenotypic plasticity for dispersal ability in the seed heteromorphic Crepis sancta (Asteraceae). - Oikos 93: 126-134.

Jongejans, E. et al. 2008. Dispersal, demography and spatial population models for conservation and control management. - Perspect. Plant Ecol. Evol. Syst. 9: 153-170.

Keane, R. M. and Crawley, M. J. 2002. Exotic plant invasions and the enemy release hypothesis. - Trends Ecol. Evol. 17: 164-170.

Kessel, B. 1953. Distribution and migration of the European starling in North America. - Condor 55: 49-67.

Korner-Nievergelt, F. et al. 2010. Improving the analysis of movement data from marked individuals through explicit estimation of observer heterogeenity. - J. Avian Biol. 41: 8-17.

Lack, D. 1968. Ecological adaptations for breeding in birds. - Methuen.

Long, J. L. 1981. Introduced birds of the world. - David and Charles.

Lowe, S. et al. 2004. 100 of the world's worst invasive alien species: a selection from the global invasive species database. - The invasive Species Specialist Group (ISSG), the Species Survival Commission (SSC) of the World Conservation Union (IUCN).

Lyons, D. A. and Scheibling, R. E. 2009. Range expansion by invasive marine algae: rates and patterns of spread at a regional scale. - Divers. Distrib. 15: 762-775.

McPeek, M. A. and Holt, R. D. 1992. The evolution of dispersal in spatially and temporally varying environments. - Am. Nat. 140: 1010-1027.

Melbourne, B. A. and Hastings, A. 2009. Highly variable spread rates in replicated biological invasions: fundamental limits to predictability. - Science 325: 1536-1539. 
Molofsky, J. and Ferdy, J. B. 2005. Extinction dynamics in experimental metapopulations. - Proc. Natl Acad. Sci. USA 102: 3726-3731.

Nathan, R. 2006. Long-distance dispersal of plants. - Science 313: 786-788.

Nathan, R. et al. 2002. Mechanisms of long-distance dispersal of seeds by wind. - Nature 418: 409-413.

Newton, I. 2004. The recent declines of farmland bird populations in Britain: an appraisal of causal factors and conservation actions. - Ibis 146: 579-600.

Nowak, M. and Sigmund, K. 1993. A strategy of win-stay, loseshift that outperforms tit-for-tat in the Prisoner's Dilemma game. - Nature 364: 56-58.

Okubo, A. 1986. Diffusion-type models for avian range expansion. - In: Ouellet, H. (ed.), Acta XIX Congressus Internationalis Ornithologici. Univ. of Ottawa Press, pp. 1038-1049.

Paradis, E. et al. 1998. Patterns of natal and breeding dispersal in birds. - J. Anim. Ecol. 67: 518-536.

Payton, M. E. et al. 2003. Overlapping confidence intervals or standard error intervals: what do they mean in terms of statistical significance? - J. Insect Sci. 3: 34.

Phillips, B. L. et al. 2006. Invasion and the evolution of speed in toads. - Nature 439: 803.

Phillips, B. L. et al. 2008. Reid's paradox revisited: the evolution of dispersal kernels during range expansion. - Am. Nat. 172: S34-S48.

Phillips, B. L. et al. 2010. Life-history evolution in range-shifting populations. - Ecology 91: 1617-1627.

Prentis, P. J. et al. 2008. Adaptive evolution in invasive species. - Trends Plant Sci. 13: 288-294.

Pulliam, H. R. 2000. On the relationship between niche and distribution. - Ecol. Lett. 3: 349-361.

Pyšek, P. and Hulme, P. E. 2005. Spatio-temporal dynamics of plant invasions: linking pattern to process. - Ecoscience 12: 302-315.

Ramos-Fernandez, G. et al. 2004. Levy walk patterns in the foraging movements of spider monkeys (Ateles geoffroyi). - Behav. Ecol. Sociobiol. 55: 223-230.

Robinson, R. A. and Sutherland, W. J. 2002. Post-war changes in arable farming and biodiversity in Great Britain. - J. Appl. Ecol. 39: 157-176.

Robinson, R. A. et al. 2005. Status and population trends of the starling Sturnus vulgaris in Great Britain. - Bird Study 52: 252-260.

Rollins, L. A. et al. 2011. Mitochondrial DNA offers unique insights into invasion history of the common starling. - Mol. Ecol. 20: 2307-2317.

Roura-Pascual, N. et al. 2011. Relative roles of climatic suitability and anthropogenic influence in determining the pattern of spread in a global invader. - Proc. Natl Acad. Sci. USA 108: 220-225.

Schoener, T. W. 2011. The newest synthesis: understanding the interplay of evolutionary and ecological dynamics. - Science 331: 426-429.

Senar, J. C. et al. 2006. Local differentiation in the presence of gene flow in the citril finch Serinus citronella. - Biol. Lett. 2: $85-87$.

Supplementary material (Appendix E7697 at <www. oikosoffice.lu.se/appendix $>$ ). Appendix 1-2.
Shigesada, N. et al. 1995. Modeling stratified diffusion in biological invasions. - Am. Nat. 146: 229-251.

Shine, R. et al. 2011. An evolutionary process that assembles phenotypes through space rather than through time. - Proc. Natl Acad. Sci. USA 108: 5708-5711.

Shlesinger, M. F. 2001. Physics in the noise. - Nature 411: 641.

Sibson, R. 1981. A brief description of natural neighbor interpolation. - In: Barnett, V. (ed.), Interpolating multivariate data. Wiley, pp. 21-36.

Simmons, A. D. and Thomas, C. D. 2004. Changes in dispersal during species' range expansions. - Am. Nat. 164: 378-395.

Skellam, J. G. 1951. Random dispersal in theoretical populations. - Biometrika 38: 196-218.

Soberon, J. 2007. Grinnellian and Eltonian niches and geographic distributions of species. - Ecol. Lett. 10: 1115-1123.

Sol, D. et al. 2002. Behavioural flexibility and invasion success in birds. - Anim. Behav. 63: 495-502.

Suarez, A. V. et al. 2001. Patterns of spread in biological invasions dominated by long-distance jump dispersal: insights from Argentine ants. - Proc. Natl Acad. Sci. USA 98: 1095-1100.

Suter, W. et al. 2009. Landscape permeability: from individual dispersal to population persistence. - In: Kienast, F. et al. (eds), A changing world: challenges for landscape research. Springer, pp. 157-174.

Travis, J. M. J. and Dytham, C. 1999. Habitat persistence, habitat availability and the evolution of dispersal. - Proc. R. Soc. B 266: 723-728.

Turchin, P. 1998. Quantitative analysis of movement: measuring and modeling population redistribution in animals and plants. - Sinauer.

Vandenbosch, F. et al. 1990. The velocity of spatial population expansion. - J. Math. Biol. 28: 529-565.

Vandenbosch, F. et al. 1992. Analyzing the velocity of animal range expansion. - J. Biogeogr. 19: 135-150.

Viswanathan, G. M. et al. 1996. Levy flight search patterns of wandering albatrosses. - Nature 381: 413-415.

Von der Lippe, M. and Kowarik, I. 2007. Long-distance dispersal of plants by vehicles as a driver of plant invasions. - Conserv. Biol. 21: 986-996.

Watson, D. 1992. Contouring: a guide to the analysis and display of spatial data. - Pergamon Press.

Wernham, C. et al. 2002. The migration atlas: movements of the birds of Britain and Ireland. - T and AD Poyser.

Whittaker, R. J. et al. 2005. Conservation biogeography: assessment and prospect. - Divers. Distrib. 11: 3-23.

Wiens, J. J. and Graham, C. H. 2005. Niche conservatism: integrating evolution, ecology, and conservation biology. - Annu. Rev. Ecol. Evol. Syst. 36: 519-539.

Williamson, M. 2009. Variation in the rate and pattern of spread in introduced species and its implications. - In: Perrings, C. et al. (eds), Bioinvasions and globalization: ecology, economics, management, and policy. Oxford Univ. Press, pp. 56-65.

Wing, L. 1943. Spread of the starling and English sparrow. - Auk 60: 74-87.

With, K. A. et al. 1999. Movement responses to patch structure in experimental fractal landscapes. - Ecology 80: 1340-1353. 